Received: 2021/10/19, Revised: 2021/10/29, Accepted: 2021/10/30, Published: 2021/12/31 @2021 Jonghoon Park et al.; Licence Physical Activity and Nutrition. This is an open access article distributed under the terms of the creative commons attribution license (https:/l creativecommons.org/licenses/by-nc/2.0/), which permits unrestricted use, distribution, and reproduction in any medium, provided the orginal work is properly cited. ${ }^{*}$ Corresponding author : Jonghoon Park, Ph.D. Exercise Nutrition and Biochemistry Laboratory, Department of Physical Education, Korea University, 145 Anamro, Seongbuk-gu, Seoul 02841, Republic of Korea

Tel: +82-2-3290-2315

E-mail: jonghoonp@korea.ac.kr

@2021 The Korean Society for Exercise Nutrition
[Purpose] This study analyzed data from the $7^{\text {th }}$ Korean National Health and Nutrition Examination Survey (2016-2018) to compare the differences in energy intake and physical activity (PA) levels in middle-aged Korean men aged $40-59$ years according to household type and the presence of metabolic syndrome (MetS).

[Methods] Data from 2,266 young adults (aged $>39$ years and $<60$ years) were obtained from the $\mathrm{KN}$ HANES. We analyzed the differences in energy intake and PA levels according to household type and MetS. The presence or absence of MetS was determined by measuring waist circumference, blood pressure, fasting blood glucose, triglyceride, and HDL-C levels.

[Results] Regarding total nutritional intake, no relationship with MetS was observed regardless of household type (interaction between MetS and household type: $p=0.875)$. No differences in total PA were observed between multiple- and single-person households (interaction between MetS and household type: $p=0.122$ ). The relationship between MetS and MetS components according to nutritional intake showed that participants with a higher energy intake had a $27 \%$ lower prevalence of low high-density lipoprotein cholesterol (HDL-C) $(p<0.05)$ and a $36 \%$ higher prevalence of high blood pressure $(p<0.01)$. The relationship between MetS and MetS components according to the PA level showed significantly reduced prevalence of MetS by approximately $39 \%, 35 \%$, and $43 \%(p<0.01,0.01$, and 0.001 ) in subjects who were somewhat active, active, and very active, respectively, compared to inactivity.

[Conclusion] Middle-aged men showed no difference in nutritional intake and PA between multiple- and single-person households. However, the risk of MetS was significantly higher in participants with approximately

1.5 times the energy intake compared to the group with the lowest energy intake. Moreover, higher levels of PA had a marked positive effect on the risk factors and prevalence of MetS. Therefore, we suggest that reducing the total energy intake and increasing total PA are important for preventing MetS in middle-aged men regardless of household type.

[Keywords] male, adults, metabolic syndrome, energy intake, physical activity, household type

\section{Comparative analysis of energy intake and physical activity according to household type and presence of metabolic syndrome in middle-aged men based on data from the $7^{\text {th }}$ Korea national health and nutrition examination survey (KNHANES) (2016-2018)}

\author{
Jonghoon Park ${ }^{1^{*} \dagger} /$ Yeonhee Park ${ }^{1 \dagger} /$ Youngjun Lee $^{1} /$ \\ Jungwoo Lee ${ }^{2}$ / Seunghee Lee ${ }^{1}$ / Chulho Shin ${ }^{3}$ / Eun-Sook Sung1 \\ 1. Department of Physical Education, Korea University, Seoul, Republic of Korea \\ 2. Department of Home Economics Education, Korea University, Seoul, Republic of Korea \\ 3. Department of Health Care, Namseoul University, Cheonan, Republic of Korea \\ $\dagger$ These two authors contributed to this work equally as the first author.
}

\section{INTRODUCTION}

In modern society, rapid economic growth and advances in science and technology have led to increased income and quality of life, along with a rapid increase in life expectancy. However, the advent of the convenience of an automated lifestyle has led to decreased physical activity (PA) and increased problems with excessive nutritional intake due to a Westernized lifestyle continue to occur. Compared to other age groups, middle-aged men report high levels of stress, drinking, and smoking, all of which are risk factors for metabolic syndrome (MetS) and lack of exercise $^{1}$.

MetS refers to the combination of the risk factors for cardiovascular disease and diabetes and is diagnosed as the combined presence of three or more of abdominal obesity, hypertriglyceridemia, low high-density lipoprotein (HDL)-cholesterol, high blood pressure, and blood sugar disorder. The major factors that increase the risk for MetS, which is the main cause of chronic diseases, are closely related to an increased incidence of obesity due to increased dietary intake and sedentary behavior ${ }^{2,3}$ In turn, the main cause of obesity is imbalanced energy intake and consumption; that is, excessive energy intake due to improper eating habits and decreased energy consumption due to lack of PA, although it may also be caused by metabolic problems or genetic factors ${ }^{4}$.

The number of single-person households is increasing rapidly worldwide due to the recent increase in divorce rates, changes in the view of marriage in younger generations, and economic poverty. In Korea, the number of single-person households as of 2019 was 6.15 million or $30.2 \%$ of the total households, making it the most typical type of household ${ }^{5}$. A study on the trends in household composition by age group from 2005 to 2015 based on population and housing census data reported a 
relatively high increase in single-person households among middle-aged men in their 40s and 50s compared to that in the young and elderly populations ${ }^{6}$. Moreover, compared to multiple-person households, single-person households are approximately two-fold more likely to lack nutritional intake based on the level recommended for Koreans ${ }^{7}$ and higher health-related risks due to a lack of $\mathrm{PA}^{8}$. In addition, a recent study of single-person households in Korea showed a higher risk of MetS in single-person households than in multiple-person households due to poorer health-related life habits ${ }^{9}$. Despite these findings, few studies have assessed the relationship between energy intake and PA level and MetS in single- and multiple-person households, and even fewer studies have been conducted in middle-aged men with a high risk of MetS. Furthermore, most of the studies on MetS, nutritional intake, and PA level according to household type have focused on the elderly population ${ }^{10-12}$, suggesting the need for more research in middle-aged men with a high risk of MetS

Therefore, this study analyzed data from the $7^{\text {th }}$ Korean National Health and Nutrition Examination Survey (KNHANES) (2016-2018) to compare the differences in energy intake and PA levels in middle-aged Korean men aged 4059 years according to household type and the presence of MetS to elucidate their relationships.

\section{METHODS}

\section{Sample and Design}

This study used cross-sectional data from the KNHANES 2016-2018 conducted by the Korea Centers for Disease Control and Prevention (KCDC). The details of the study design and data resource profiles followed the methods in the Guidelines for Use of the KNHANES Raw Data and the Final Report of the sampling frame ${ }^{13}$. The KNHANES consists of a health interview survey, a nutrition survey, and a health examination, and was conducted according to the principles of the Declaration of Helsinki. This survey was approved by the Institutional Review Board of the Korea Centers for Disease Control and Prevention (reference number: 2018-01-03-P-A). All participants signed an informed consent form. Between 2016 and 2018, 24,269 individuals completed a health interview survey, nutrition survey, and health examination. Among them, 17,020 participants aged $<39$ or $>60$ years were excluded and 7,249 aged between 40 and 59 years remained. A total of 4,056 middle-aged women

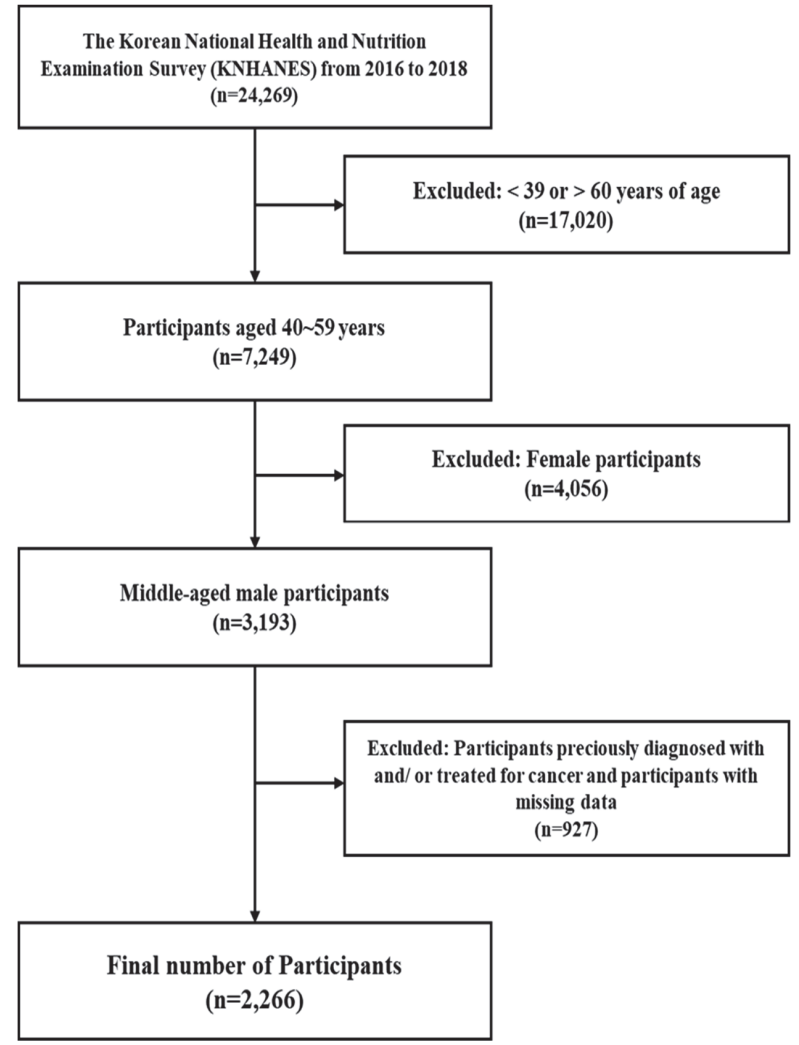

Figure 1. Flow diagram of the study participants.

were excluded. Finally, 3,193 participants were included. Participants previously diagnosed with cancer (gastric, liver, colon, breast, cervical, lung, thyroid, and other cancers) and those with missing data (anthropometric, health examination, and PA) were excluded (Figure 1). Thus, this study included 2,266 middle-aged adults

\section{Measures}

The presence or absence of MetS was determined by measuring waist circumference, blood pressure, fasting blood glucose levels, triglyceride levels, and HDL-C levels. PA variables were evaluated using the Global Physical Activity Questionnaire (GPAQ), and PA was expressed in metabolic equivalents (MET)-minutes/week. Nutrient intake was also assessed. The characteristics of the participants according to the household are shown in Table 1.

Table 1. Participant characteristics by household type.

\begin{tabular}{|c|c|c|c|c|c|c|c|c|c|}
\hline \multirow[b]{2}{*}{ Variables } & \multicolumn{3}{|c|}{ Total $(n=2,266)$} & \multicolumn{3}{|c|}{ Multiple $(n=2,057)$} & \multicolumn{3}{|c|}{ Single $(n=209)$} \\
\hline & $\begin{array}{l}\text { Non-MetS } \\
(n=1,454)\end{array}$ & $\begin{array}{c}\text { MetS } \\
(n=812)\end{array}$ & $p$--Value & $\begin{array}{l}\text { Non-MetS } \\
(n=1,317)\end{array}$ & $\begin{array}{c}\text { MetS } \\
(n=740)\end{array}$ & $p$-Value & $\begin{array}{c}\text { Non-MetS } \\
(n=137)\end{array}$ & $\begin{array}{l}\text { MetS } \\
(n=72)\end{array}$ & $p$-Value \\
\hline Age (years) & $49.4 \pm 0.2$ & $49.7 \pm 0.2$ & 0.276 & $49.4 \pm 0.2$ & $49.7 \pm 0.2$ & 0.323 & $49.3 \pm 0.5$ & $49.7 \pm 0.7$ & 0.615 \\
\hline Height (cm) & $170.7 \pm 0.2$ & $171.5 \pm 0.2$ & 0.006 & $170.8 \pm 0.2$ & $171.5 \pm 0.2$ & 0.018 & $169.3 \pm 0.6$ & $171.0 \pm 0.8$ & 0.090 \\
\hline Body weight (kg) & $69.0 \pm 0.3$ & $78.2 \pm 0.4$ & $<0.001$ & $69.3 \pm 0.3$ & $78.3 \pm 0.4$ & $<0.001$ & $65.8 \pm 0.7$ & $77.2 \pm 1.6$ & $<0.001$ \\
\hline $\mathrm{BMI}\left(\mathrm{kg} / \mathrm{m}^{2}\right)$ & $23.7 \pm 0.1$ & $26.6 \pm 0.1$ & $<0.001$ & $23.7 \pm 0.1$ & $26.6 \pm 0.1$ & $<0.001$ & $23.0 \pm 0.2$ & $26.4 \pm 0.5$ & $<0.001$ \\
\hline MetS (\%) & $65.0 \pm 1.2$ & $35.0 \pm 1.2$ & $<0.001$ & $64.7 \pm 1.3$ & $35.3 \pm 1.3$ & $<0.001$ & $68.7 \pm 3.8$ & $31.3 \pm 3.8$ & $<0.001$ \\
\hline
\end{tabular}

Data are expressed as the mean \pm standard error. MetS, metabolic syndrome; BMI, body mass index. 


\section{Energy Intake}

The nutrition survey of the KNHANES consisted of a survey of dietary habits, one-day $24 \mathrm{~h}$ recall, and a food frequency questionnaire. The nutrition survey data were collected by trained dietitians in the participants' homes one week after the health interview and health examination. Daily energy intake was calculated using the Korean Foods and Nutrients Database of the Rural Development Administration. The analyses included the following items: total energy intake, carbohydrate intake, protein intake, and fat intake. Energy intake was categorized by dividing the ratio by the estimated energy requirement (EER). Values $<20 \%$ of the EER $(<0.8)$ were considered lower intake, while values above 1.2 were considered high intake.

\section{PA}

The GPAQ comprises 16 questions grouped to capture PA performed in different behavioral domains, namely, work, transport, and discretionary (leisure or recreational) activities. It analyzes five domains of PA: vigorous-intensity work, moderate-intensity work, place movement, vigorous-intensity recreation, and moderate-intensity recreation. The World Health Organization (WHO) GPAQ analysis guidelines were used to analyze GPAQ data ${ }^{14}$. We estimated that, compared to sitting quietly, an individual's caloric consumption was four and eight times higher while moderately and vigorously active, respectively. Therefore, when calculating the total energy consumption of an individual using GPAQ data, four and eight METs were allocated to the time spent in moderate and vigorous activity, respectively, defined as follows:

- Vigorous-intensity activity: occupational (MET) $=8.0$ $\times$ vigorous-intensity PA (day/week) $\times$ one-day vigorous-intensity PA (min/day)

- Moderate-intensity activity: occupational $(\mathrm{MET})=4.0$ $\times$ moderate-intensity PA $($ day $/$ week $) \times$ one-day moderate-intensity PA (min/day)

- Vigorous-intensity activity: recreational $(\mathrm{MET})=8.0$ $\times$ vigorous-intensity PA (day/week) $\times$ one-day vigorous-intensity physical activity ( $\mathrm{min} /$ day)

- Moderate-intensity activity: recreational $(\mathrm{MET})=4.0$ $\times$ moderate-intensity PA $($ day $/$ week $) \times$ one-day moderate-intensity PA (min/day)

- Place movement $(\mathrm{MET})=4.0 \times$ place movement PA $($ day/week $) \times$ one-day place movement PA

- Total PA (MET) = vigorous-intensity activity: occupational + moderate-intensity activity; occupational + vigorous-intensity activity; recreational + moderate-intensity activity; recreational + place movement.

PA levels were classified into four groups: inactive (0$249 \mathrm{MET} \mathrm{min} /$ week), somewhat active (250-499 MET min/ week), active (500-999 MET min/week), and very active ( $>1000$ MET min/week). These cut-off points are based on their equivalence to the following PA thresholds: 250 MET $\mathrm{min} /$ week represents an energy expenditure dose equivalent to half of the threshold, $500 \mathrm{MET} \mathrm{min} /$ week is equivalent to the minimum threshold, and $1000 \mathrm{MET}$ min/week is equivalent to twice the minimum threshold.

\section{Statistical Analysis}

Continuous variables were presented as means and standard errors. The normality of the distributions of all outcome variables was verified using the Kolmogorov-Smirnov test. Independent $t$-tests were used to analyze the risk factors for MetS, PA levels, and energy intake between the non-MetS and MetS groups. Two-way analysis of variance (ANOVA) was used to analyze the differences in risk factors for MetS, PA levels, energy intake between participants with and without MetS, and between single-person and multi-person households. Partial eta-squared $\left(\eta^{2}\right)$ values were calculated as measures of the effect size. If a significant interaction effect was found in the two-way ANOVA, Bonferroni post hoc tests were used to compare the presence and absence of MetS and the household-specificity of dependent variables in each group separately. Moreover, the relationships between PA levels or energy intake and MetS were determined using logistic regression after controlling for covariates. Logistic regression findings were presented as odds ratios (ORs) and their associated $95 \%$ confidence intervals (CIs). Statistical analyses were performed using IBM SPSS Statistics for Windows (version 25.0; IBM Corp., Armonk, NY, USA), with $p<0.05$, considered statistically significant.

\section{RESULTS}

\section{Comparisons of risk factors for MetS by household type according to the presence of MetS}

Table 2 presents the risk factors for MetS according to the presence of MetS by household type in middle-aged men. Analysis of the differences in the risk factors of MetS for the entire population showed significantly higher waist circumference, triglyceride, systolic blood pressure, diastolic blood pressure, fasting blood glucose, and glycated hemoglobin values for the group with MetS compared to those in the group without MetS (all $p<0.001$ ). HDL cholesterol was significantly lower in the group with MetS than in the group without MetS $(p<0.001)$.

Analysis of the differences in risk factors of MetS by household type for the entire population revealed a significant interaction effect in triglycerides and fasting blood glucose by household type and the presence of MetS $(p<0.001)$. In the subsequent post-hoc test using Bonferroni correction, triglyceride levels were significantly higher in single-person households than in multi-person households in the group with MetS $(p<0.01)$. The fasting blood glucose level was significantly higher in single-person households than in multi-person households, regardless of the presence of MetS $(p<0.01)$.

\section{Energy intake by household type according to the presence of MetS}

Table 3 shows the differences in energy intake by household type according to the presence of MetS in middle-aged men. Analysis of the difference in energy intake for the entire population showed significantly lower carbohydrate intake in the group with MetS than in the group without MetS 
Table 2. Metabolic syndrome components.

\begin{tabular}{|c|c|c|c|c|c|c|c|c|}
\hline \multirow{2}{*}{ Variables } & \multirow{2}{*}{ Group } & \multirow{2}{*}{ Total } & \multirow{2}{*}{ Multiple } & \multirow{2}{*}{ Single } & \multicolumn{4}{|c|}{ ANOVA } \\
\hline & & & & & \multicolumn{2}{|c|}{ F-value } & $p$-Value $\left(\eta^{2}\right)$ & Power \\
\hline \multirow{3}{*}{ Waist (cm) } & Non-MetS & $83.6 \pm 0.2$ & $83.8 \pm 0.2$ & $81.9 \pm 0.6$ & $\mathrm{H}$ & 2.870 & $0.090(0.000)$ & 0.395 \\
\hline & MetS & $92.2 \pm 0.3$ & $92.3 \pm 0.3$ & $91.1 \pm 1.4$ & M & 93.493 & $0.000(0.004)$ & 1.000 \\
\hline & $p$-value & $<0.001$ & & & $\mathrm{H} \times \mathrm{M}$ & 0.182 & $0.670(0.000)$ & 0.071 \\
\hline \multirow{3}{*}{$\mathrm{TG}(\mathrm{mg} / \mathrm{dL})$} & Non-MetS & $136.3 \pm 2.9$ & $134.7 \pm 2.6$ & $152.3 \pm 19.5$ & $\mathrm{H}$ & 40.971 & $0.000(0.002)$ & 1.000 \\
\hline & MetS & $274.0 \pm 7.5$ & $267.3 \pm 7.0 \dagger$ & $355.1 \pm 48.5 \dagger \ddagger$ & $M$ & 415.635 & $0.000(0.021)$ & 1.000 \\
\hline & $p$-value & $<0.001$ & & & $\mathrm{H} \times \mathrm{M}$ & 18.200 & $0.000(0.001)$ & 0.989 \\
\hline \multirow{3}{*}{ HDL-C (mg/dL) } & Non-MetS & $49.5 \pm 0.3$ & $49.4 \pm 0.3$ & $50.8 \pm 1.3$ & $\mathrm{H}$ & 1.691 & $0.193(0.000)$ & 0.255 \\
\hline & MetS & $41.8 \pm 0.4$ & $41.7 \pm 0.4$ & $42.6 \pm 1.7$ & $M$ & 80.587 & $0.000(0.004)$ & 1.000 \\
\hline & $p$-value & $<0.001$ & & & $\mathrm{H} \times \mathrm{M}$ & 0.102 & $0.749(0.000)$ & 0.062 \\
\hline \multirow{3}{*}{$\mathrm{SBP}(\mathrm{mmHg})$} & Non-MetS & $116.6 \pm 0.4$ & $116.3 \pm 0.4$ & $119.8 \pm 1.4$ & $\mathrm{H}$ & 7.549 & $0.006(0.000)$ & 0.784 \\
\hline & MetS & $127.6 \pm 0.6$ & $127.4 \pm 0.6$ & $130.1 \pm 2.1$ & $M$ & 90.618 & $0.000(0.004)$ & 1.000 \\
\hline & $p$-value & $<0.001$ & & & $\mathrm{H} \times \mathrm{M}$ & 0.141 & $0.707(0.000)$ & 0.066 \\
\hline \multirow{3}{*}{$\mathrm{DBP}(\mathrm{mmHg})$} & Non-MetS & $78.7 \pm 0.2$ & $78.6 \pm 0.2$ & $79.5 \pm 0.8$ & $\mathrm{H}$ & 3.025 & $0.082(0.000)$ & 0.413 \\
\hline & MetS & $86.8 \pm 0.4$ & $86.6 \pm 0.4$ & $88.3 \pm 1.6$ & $\mathrm{M}$ & 135.190 & $0.000(0.007)$ & 1.000 \\
\hline & $p$-value & $<0.001$ & & & $\mathrm{H} \times \mathrm{M}$ & 0.356 & $0.356(0.000)$ & 0.092 \\
\hline \multirow{3}{*}{ Glucose (mg/dL) } & Non-MetS & $99.3 \pm 0.6$ & $98.9 \pm 0.5$ & 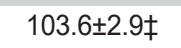 & $\mathrm{H}$ & 47.468 & $0.000(0.002)$ & 1.000 \\
\hline & MetS & $116.9 \pm 1.2$ & $115.6 \pm 1.1 \dagger$ & 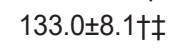 & $\mathrm{M}$ & 204.489 & $0.000(0.010)$ & 1.000 \\
\hline & p-value & $<0.001$ & & & $\mathrm{H} \times \mathrm{M}$ & 15.587 & $0.000(0.001)$ & 0.977 \\
\hline \multirow{3}{*}{ HbAlc (\%) } & Non-MetS & $5.6 \pm 0.0$ & $5.6 \pm 0.0$ & $5.7 \pm 0.1$ & $\mathrm{H}$ & 16.246 & $0.000(0.001)$ & 0.981 \\
\hline & MetS & $6.0 \pm 0.0$ & $6.0 \pm 0.0$ & $6.3 \pm 0.2$ & $\mathrm{M}$ & 76.994 & $0.000(0.004)$ & 1.000 \\
\hline & $p$-value & $<0.001$ & & & $\mathrm{H} \times \mathrm{M}$ & 2.867 & $0.090(0.000)$ & 0.395 \\
\hline
\end{tabular}

Data are expressed as the mean \pm standard error. ANOVA, analysis of variance; MetS, metabolic syndrome; TG, triglycerides; HDL-C, high-density lipoprotein cholesterol; SBP, systolic blood pressure; DBP, diastolic blood pressure; HbA1c, glycated hemoglobin.

Main effect $=\mathrm{H}$ (household) and M (metabolic syndrome), Interaction effect $=\mathrm{H} \times \mathrm{M}$ (household $\times$ metabolic syndrome), $\dagger p<0.001$ vs. non-MetS values, $\ddagger p<0.01$ vs. multiple values. Two-way ANOVA followed by Bonferroni post hoc tests.

Table 3. Energy intake.

\begin{tabular}{|c|c|c|c|c|c|c|c|c|}
\hline \multirow{2}{*}{ Variables } & \multirow{2}{*}{ Group } & \multirow{2}{*}{ Total } & \multirow{2}{*}{ Multiple } & \multirow{2}{*}{ Single } & \multicolumn{4}{|c|}{ ANOVA } \\
\hline & & & & & & llue & $p$-Value $\left(\eta^{2}\right)$ & Power \\
\hline \multirow{4}{*}{$\begin{array}{l}\text { Total } \\
\text { Energy intake } \\
\text { (kcal) }\end{array}$} & Non-MetS & $2464.1 \pm 30.2$ & $2466.6 \pm 32.0$ & $2438.3 \pm 93.1$ & $\mathrm{H}$ & 0.091 & $0.763(0.000)$ & 0.060 \\
\hline & & & & & & & & \\
\hline & MetS & $2471.6 \pm 40.4$ & $2472.2 \pm 39.5$ & $2463.3 \pm 169.8$ & M & 0.061 & $0.804(0.000)$ & 0.057 \\
\hline & $p$-value & 0.884 & & & $\mathrm{H} \times \mathrm{M}$ & 0.025 & $0.875(0.000)$ & 0.053 \\
\hline \multirow{3}{*}{$\begin{array}{l}\text { Carbohydrate } \\
\text { intake }(g)\end{array}$} & Non-MetS & $351.3 \pm 4.1$ & $353.0 \pm 4.2$ & $333.6 \pm 11.5$ & $\mathrm{H}$ & 3.751 & $0.053(0.000)$ & 0.491 \\
\hline & MetS & $330.4 \pm 4.8$ & $331.4 \pm 5.0$ & $317.9 \pm 18.4$ & M & 4.798 & $0.029(0.000)$ & 0.591 \\
\hline & $p$-value & 0.001 & & & $\mathrm{H} \times \mathrm{M}$ & 0.123 & $0.726(0.000)$ & 0.064 \\
\hline \multirow{3}{*}{ Fat intake (g) } & Non-MetS & $54.1 \pm 1.2$ & $54.3 \pm 1.2$ & $52.4 \pm 4.1$ & $\mathrm{H}$ & 1.803 & $0.179(0.000)$ & 0.269 \\
\hline & MetS & $53.3 \pm 1.5$ & $53.7 \pm 1.6$ & $49.1 \pm 5.0$ & M & 0.637 & $0.425(0.000)$ & 0.126 \\
\hline & $p$-value & 0.672 & & & $\mathrm{H} \times \mathrm{M}$ & 0.311 & $0.577(0.000)$ & 0.086 \\
\hline \multirow{3}{*}{ Protein intake (g) } & Non-MetS & $87.9 \pm 1.3$ & $88.1 \pm 1.4$ & $85.1 \pm 4.8$ & $\mathrm{H}$ & 0.524 & $0.469(0.000)$ & 0.112 \\
\hline & MetS & $85.6 \pm 1.5$ & $85.6 \pm 1.5$ & $84.7 \pm 6.4$ & $M$ & 0.265 & $0.607(0.000)$ & 0.081 \\
\hline & $p$-value & 0.237 & & & $\mathrm{H} \times \mathrm{M}$ & 0.155 & $0.694(0.000)$ & 0.068 \\
\hline
\end{tabular}

Data are expressed as the mean \pm standard error. ANOVA, analysis of variance; MetS, metabolic syndrome.

Main effect $=\mathrm{H}$ (household) and $\mathrm{M}$ (metabolic syndrome), Interaction effect $=\mathrm{H} \times \mathrm{M}$ (household $\times$ metabolic syndrome), $† p<0.001$ vs. non-MetS values, $\ddagger p<0.05$ vs. multiple values. Two-way ANOVA followed by Bonferroni post hoc tests. 
( $p=0.001)$, and no differences between the two groups in other categories ( $p=0.884,0.672$, and 0.237 in the total energy intake, fat intake, and protein intake, respectively).

Analysis of the difference in energy intake by household type in the entire population showed no significant interaction effects by household type and MetS for all categories ( $p=0.875,0.726,0.577$, and 0.694 for total energy intake, carbohydrate, fat, and protein intakes, respectively).

\section{PA levels by household type according to the pres- ence of MetS}

Table 4 shows the differences in PA levels by household type according to the presence of MetS in middle-aged men. When the difference in PA level was analyzed for the entire population, the group with MetS showed significantly lower values in recreational vigorous and moderate than the group without MetS $(p<0.001$ and $p 0.05$ in recreational vigorous and moderate, respectively), while there were no differences between the groups for other categories $(p=0.612,0.717$, 0.327 , and 0.292 in occupational vigorous, occupational moderate, place movement, and total physical activity, respectively).

Analysis of the difference in PA level by household type for the entire population showed a significant interaction effect by household type and the presence of MetS for occupational vigorous and recreational moderate of $p=0.002$ and 0.002 , respectively). A subsequent post hoc test with Bonferroni correction showed a significantly higher level of occupational vigorous in single-person households than that of multi-person households in the group with MetS ( $p$
$<0.01)$. For recreational moderate, the group with MetS showed significantly lower values than those without MetS in multiple-person households $(p<0.001)$. In contrast, in single-person households, the group with MetS showed significantly higher values than those without MetS $(p<0.001)$. However, there was no difference in the total PA by household type and presence of MetS (Interaction: $p=0.122$ ).

\section{ORs of MetS and MetS components according to} energy intake and level of PA (95\% CIs)

Examination of the relationship between the actual energy intake based on the EER (energy intake/EER) and MetS showed no relationship between the prevalence of MetS and energy intake/EER. However, low HDC-cholesterol level was significantly decreased for high energy intake (OR = $0.73,95 \% \mathrm{CI}=0.54-1.00, p<0.05$ ) based on a lower energy intake/EER. High blood pressure was also significantly increased for high energy intake $(\mathrm{OR}=1.36,95 \% \mathrm{CI}=1.10$ $1.68, p<0.01)$. High triglyceride levels were decreased for moderate energy intake/EER $(\mathrm{OR}=0.78,95 \% \mathrm{CI}=0.63$ $0.95, p<0.05$ ), but no relationship was observed for a higher energy intake/EER.

Table 5 shows the mean ratio of actual nutritional intake based on the mean PA level according to PA level and EER. The results of the complex sample logistic regression analysis of the relationship between MetS and MetS components according to PA level and nutritional intake are shown in Table 6. This model was corrected for body mass index (BMI) (Table 1) and household type and showed differences according to the presence of MetS. The prevalence of MetS

Table 4. Physical activity levels.

\begin{tabular}{|c|c|c|c|c|c|c|c|c|}
\hline \multirow{2}{*}{$\begin{array}{l}\text { Physical Activity } \\
\text { (MET × } \mathrm{min} / \text { Week) }\end{array}$} & \multirow{2}{*}{ Group } & \multirow{2}{*}{ Total } & \multirow{2}{*}{ Multiple } & \multirow{2}{*}{ Single } & \multicolumn{4}{|c|}{ ANOVA } \\
\hline & & & & & & & $p$-Value $\left(\eta^{2}\right)$ & Power \\
\hline \multirow{3}{*}{$\begin{array}{l}\text { Occupational } \\
\text { vigorous }\end{array}$} & Non-MetS & $99.6 \pm 36.2$ & $104.4 \pm 39.5$ & $52.0 \pm 36.1$ & $\mathrm{H}$ & 4.000 & $0.046(0.000)$ & 0.516 \\
\hline & MetS & $130.7 \pm 49.7$ & $111.1 \pm 44.4$ & $367.6 \pm 361.3 † \dagger \ddagger$ & M & 9.973 & $0.002(0.000)$ & 0.885 \\
\hline & $p$-value & 0.612 & & & $\mathrm{H} \times \mathrm{M}$ & 9.155 & $0.002(0.000)$ & 0.857 \\
\hline \multirow{3}{*}{$\begin{array}{l}\text { Occupational } \\
\text { moderate }\end{array}$} & Non-MetS & $161.8 \pm 27.3$ & $166.2 \pm 29.9$ & $117.5 \pm 45.6$ & $\mathrm{H}$ & 5.556 & $0.018(0.000)$ & 0.654 \\
\hline & MetS & $178.9 \pm 38.7$ & $192.0 \pm 41.6$ & $20.5 \pm 13.2$ & M & 0.582 & $0.445(0.000)$ & 0.119 \\
\hline & $p$-value & 0.717 & & & $\mathrm{H} \times \mathrm{M}$ & 1.728 & $0.189(0.000)$ & 0.260 \\
\hline \multirow{3}{*}{ Place movement } & Non-MetS & $384.3 \pm 19.1$ & $379.8 \pm 19.9$ & $430.1 \pm 71.9$ & H & 2.119 & $0.145(0.000)$ & 0.307 \\
\hline & MetS & $351.1 \pm 28.5$ & $344.1 \pm 29.7$ & $435.7 \pm 86.0$ & M & 0.095 & $0.758(0.000)$ & 0.061 \\
\hline & $p$-value & 0.327 & & & $\mathrm{H} \times \mathrm{M}$ & 0.180 & $0.671(0.000)$ & 0.071 \\
\hline \multirow{3}{*}{$\begin{array}{l}\text { Recreational } \\
\text { vigorous }\end{array}$} & Non-MetS & $215.8 \pm 19.8$ & $219.1 \pm 21.8$ & $183.1 \pm 47.1$ & $\mathrm{H}$ & 2.077 & $0.150(0.000)$ & 0.302 \\
\hline & MetS & $117.6 \pm 17.3$ & $123.3 \pm 18.7$ & $49.4 \pm 29.5$ & M & 9.057 & $0.003(0.000)$ & 0.853 \\
\hline & $p$-value & $<0.001$ & & & $\mathrm{H} \times \mathrm{M}$ & 0.248 & $0.619(0.000)$ & 0.079 \\
\hline \multirow{3}{*}{$\begin{array}{l}\text { Recreational } \\
\text { moderate }\end{array}$} & Non-MetS & $190.4 \pm 12.2$ & $199.0 \pm 13.0$ & $103.6 \pm 23.4 \ddagger$ & $\mathrm{H}$ & 0.250 & $0.617(0.000)$ & 0.079 \\
\hline & MetS & $153.0 \pm 13.6$ & $147.7 \pm 13.2 \dagger$ & $216.6 \pm 72.9 \dagger$ & M & 1.351 & $0.245(0.000)$ & 0.213 \\
\hline & $p$-value & 0.041 & & & $\mathrm{H} \times \mathrm{M}$ & 9.551 & $0.002(0.000)$ & 0.871 \\
\hline \multirow{3}{*}{$\begin{array}{l}\text { Total physical } \\
\text { activity }\end{array}$} & Non-MetS & $1052.0 \pm 67.5$ & $1068.4 \pm 73.6$ & $886.3 \pm 120.9$ & $\mathrm{H}$ & 0.002 & $0.963(0.000)$ & 0.050 \\
\hline & MetS & $931.3 \pm 92.0$ & $918.2 \pm 94.6$ & $1089.8 \pm 367.8$ & M & 0.054 & $0.816(0.000)$ & 0.056 \\
\hline & $p$-value & 0.292 & & & $\mathrm{H} \times \mathrm{M}$ & 2.397 & $0.122(0.000)$ & 0.340 \\
\hline
\end{tabular}

Data are expressed as the mean \pm standard error. ANOVA, analysis of variance; MetS, metabolic syndrome Main effect $=\mathrm{H}$ (household) and $\mathrm{M}$ (metabolic syndrome), Interaction effect $=\mathrm{H} \times \mathrm{M}$ (household $\times$ metabolic syndrome), $\uparrow p<0.001$ vs. non-MetS values, $\ddagger p<0.01$ vs. multiple values. Two-way ANOVA followed by Bonferroni post hoc tests. 
Table 5. Classification of physical activity levels and energy intake.

\begin{tabular}{|c|c|c|c|c|c|c|}
\hline Factors & Total & $\mathrm{n}$ & $\begin{array}{l}\text { Multi-person } \\
\text { Households }\end{array}$ & $n$ & $\begin{array}{l}\text { Single-person } \\
\text { Households }\end{array}$ & $\mathbf{n}$ \\
\hline Physical activity factors & \multicolumn{6}{|c|}{ MET min/Week (MeanıSE) } \\
\hline Inactive & $32.98 \pm 2.32$ & 1052 & $32.52 \pm 2.49$ & 961 & $38.10 \pm 7.95$ & 91 \\
\hline Somewhat active & $437.55 \pm 6.03$ & 255 & $435.74 \pm 6.47$ & 229 & $456.73 \pm 16.03$ & 26 \\
\hline Active & $822.98 \pm 7.98$ & 380 & $813.09 \pm 8.61$ & 336 & $916.00 \pm 30.78$ & 44 \\
\hline Very active & $3026.79 \pm 163.81$ & 579 & $3020.09 \pm 167.62$ & 531 & $3108.19 \pm 567.51$ & 48 \\
\hline Energy intake factors & \multicolumn{6}{|c|}{ Energy intake/EER (Mean $\pm S E$ ) } \\
\hline $\begin{array}{c}\text { Lower energy intake } \\
\text { (energy intake/EER<0.8) }\end{array}$ & $0.57 \pm 0.00$ & 892 & $0.60 \pm 0.01$ & 811 & $0.57 \pm 0.02$ & 81 \\
\hline $\begin{array}{c}\text { Moderate energy intake }(0.8 \leq \\
\text { energy intake/EER } \leq 1.2)\end{array}$ & $0.97 \pm 0.00$ & 845 & $0.99 \pm 0.00$ & 769 & $0.96 \pm 0.01$ & 76 \\
\hline $\begin{array}{l}\text { Higher energy intake } \\
\text { (energy intake/EER>1.2) }\end{array}$ & $1.49 \pm 0.01$ & 529 & $1.53 \pm 0.02$ & 477 & $1.58 \pm 0.06$ & 52 \\
\hline
\end{tabular}

Values are expressed as the mean \pm standard error. EER = estimated energy requirement; MET = metabolic equivalents.

Table 6. Odds ratios $(95 \% \mathrm{Cl})$ for MetS and MetS components according to physical activity levels and energy intake.

\begin{tabular}{|c|c|c|c|c|c|c|}
\hline Factors & MetS & $\begin{array}{l}\text { Large Waist } \\
\text { Circumference }\end{array}$ & $\begin{array}{l}\text { High Tri- } \\
\text { glycerides }\end{array}$ & Low HDL-C & $\begin{array}{l}\text { High Blood } \\
\text { Pressure }\end{array}$ & $\begin{array}{l}\text { High } \\
\text { Glucose }\end{array}$ \\
\hline \multicolumn{7}{|l|}{ Physical activity factors } \\
\hline Inactive $(n=1354)$ & $\begin{array}{c}1.00 \\
\text { (reference) }\end{array}$ & $\begin{array}{c}1.00 \\
\text { (reference) }\end{array}$ & $\begin{array}{c}1.00 \\
\text { (reference) }\end{array}$ & $\begin{array}{c}1.00 \\
\text { (reference) }\end{array}$ & $\begin{array}{c}1.00 \\
\text { (reference) }\end{array}$ & $\begin{array}{c}1.00 \\
\text { (reference) }\end{array}$ \\
\hline Somewhat active $(n=464)$ & $\begin{array}{c}0.61 \\
(0.43-0.85)^{\star *}\end{array}$ & $\begin{array}{c}0.61 \\
(0.37-1.00)^{*}\end{array}$ & $\begin{array}{c}0.82 \\
(0.63-1.08)\end{array}$ & $\begin{array}{c}0.81 \\
(0.58-1.12)\end{array}$ & $\begin{array}{c}0.85 \\
(0.61-1.19)\end{array}$ & $\begin{array}{c}1.03 \\
(0.76-1.40)\end{array}$ \\
\hline Active (n=834) & $\begin{array}{c}0.65 \\
(0.47-0.89)^{* *}\end{array}$ & $\begin{array}{c}0.69 \\
(0.48-1.00)^{*}\end{array}$ & $\begin{array}{c}0.93 \\
(0.69-1.25)\end{array}$ & $\begin{array}{c}0.66 \\
(0.50-0.88)^{* *}\end{array}$ & $\begin{array}{c}0.92 \\
(0.70-1.21)\end{array}$ & $\begin{array}{c}0.77 \\
(0.59-1.01)\end{array}$ \\
\hline Very active $(n=1322)$ & $\begin{array}{c}0.57 \\
(0.43-0.75)^{\star \star *}\end{array}$ & $\begin{array}{c}0.47 \\
(0.33-0.68)^{\star * *}\end{array}$ & $\begin{array}{c}0.82 \\
(0.64-1.05)\end{array}$ & $\begin{array}{c}0.62 \\
(0.49-0.78)^{\star \star *}\end{array}$ & $\begin{array}{c}0.80 \\
(0.63-1.01)\end{array}$ & $\begin{array}{c}0.85 \\
(0.68-1.07)\end{array}$ \\
\hline \multicolumn{7}{|l|}{ Energy intake factors } \\
\hline $\begin{array}{c}\text { Lower energy intake } \\
\text { (energy intake/EER<0.8) }\end{array}$ & $\begin{array}{c}1.00 \\
\text { (reference) }\end{array}$ & $\begin{array}{c}1.00 \\
\text { (reference) }\end{array}$ & $\begin{array}{c}1.00 \\
\text { (reference) }\end{array}$ & $\begin{array}{c}1.00 \\
\text { (reference) }\end{array}$ & $\begin{array}{c}1.00 \\
\text { (reference) }\end{array}$ & $\begin{array}{c}1.00 \\
\text { (reference) }\end{array}$ \\
\hline $\begin{array}{c}\text { Moderate energy intake }(0.8 \leq \\
\text { energy intake/EER } \leq 1.2)\end{array}$ & $\begin{array}{c}0.92 \\
(0.74-1.16)\end{array}$ & $\begin{array}{c}1.10 \\
(0.78-1.54)\end{array}$ & $\begin{array}{c}0.78 \\
(0.63-0.95)^{*}\end{array}$ & $\begin{array}{c}1.06 \\
(0.86-1.31)\end{array}$ & $\begin{array}{c}0.96 \\
(0.78-1.19)\end{array}$ & $\begin{array}{c}0.94 \\
(0.76-1.16)\end{array}$ \\
\hline $\begin{array}{c}\text { Higher energy intake } \\
\text { (energy intake/EER >1.2) }\end{array}$ & $\begin{array}{c}1.16 \\
(0.87-1.54)\end{array}$ & $\begin{array}{c}1.35 \\
(0.89-2.05)\end{array}$ & $\begin{array}{c}0.98 \\
(0.78-1.25)\end{array}$ & $\begin{array}{c}0.73 \\
(0.54-1.00)^{*}\end{array}$ & $\begin{array}{c}1.36 \\
(1.10-1.68)^{\star *}\end{array}$ & $\begin{array}{c}1.12 \\
(0.89-1.41)\end{array}$ \\
\hline
\end{tabular}

Data presented as odds ratios (95\% confidence intervals [Cls]). OR, odds ratio; MetS, metabolic syndrome; HDL-C, high-density lipoprotein cholesterol; EER, estimated energy requirement ${ }^{*} p<0.05$ vs. reference, ${ }^{* *} p<0.01$ vs. reference, ${ }^{* *} p<0.001$ vs. reference. The model was adjusted for household type and body mass index.

was significantly lower than the baseline values for a somewhat active PA level (somewhat active, OR $=0.61,95 \% \mathrm{CI}$ $=0.43-0.85, p<0.01 ;$ active, $\mathrm{OR}=0.65,95 \% \mathrm{CI}=0.47-0.89$, $p<0.01$; very active, $\mathrm{OR}=0.57,95 \% \mathrm{CI}=0.43-0.75, p<$ $0.001)$. Abdominal obesity was also significantly lower than the baseline values from somewhat active PA level (somewhat active, $\mathrm{OR}=0.61,95 \% \mathrm{CI}=0.37-1.00, p<0.05$; Active, $\mathrm{OR}=0.69,95 \% \mathrm{CI}=0.45-1.00, p<0.05$; Very active, $\mathrm{OR}=0.47,95 \% \mathrm{CI}=0.33-0.68, p<0.001)$. Low HDC-cholesterol levels were significantly reduced for active $(\mathrm{OR}=$ $0.66,95 \% \mathrm{CI}=0.50-0.88, p<0.01)$ and very active $(\mathrm{OR}=$ $0.62,95 \% \mathrm{CI}=0.49-0.78, p<0.001)$ levels of PA.

\section{DISCUSSION}

This study analyzed raw data from years $1-3$ of the $7^{\text {th }}$ KNHANES (2016-2018) to compare the differences in energy intake and PA levels in middle-aged Korean men aged 40-59 years according to household type and the presence of MetS. The major findings of this study are as follows: Regarding nutritional intake, there was no relationship between household type and MetS. However, the results of a complex sample logistic regression analysis to identify the relationship between MetS and MetS components according to nutritional intake showed a significantly increased risk of increased HDL cholesterol level and hypertension for low nutritional intake for a ratio of actual nutritional intake based on EER of at least 1.2. There was no difference in PA 
levels between multiple- and single-person households. The results of complex sample logistic regression analysis of the relationship between MetS and MetS components according to PA level showed a significantly reduced prevalence of MetS and abdominal obesity compared to the baseline values for the somewhat active level of PA. Moreover, the low HDL cholesterol level was significantly lower than the baseline value for active PA levels.

Analysis of the risk factors according to the presence of MetS by household type showed higher triglyceride and fasting blood sugar levels in the single-person household group with MetS compared to those in the multi-person household group. Thus, our study result based on our present data does not agree with this because there were no differences in energy intake and PA level between the single and multi-person household groups. $\mathrm{Kim}^{16}$ reported that single-person households are more likely to skip breakfast, eat out more than once a day, and eat every meal alone compared to multi-person households. This study suggests that the eating habits of single-person households are generally poorer than those of multiple-person households, thereby resulting in a higher presence of in this study. A previous study reported higher fasting blood glucose levels among cases of hyperglycemia in single-person households ${ }^{9}$, while a study of middle-aged and elderly subjects reported higher triglyceride and fasting blood glucose levels in single-person households than in multi-person households ${ }^{15}$. Therefore, the prevalence of hyperlipidemia and diabetes may be higher in middle-aged men with MetS in single-person households.

The middle-aged men in this study showed no difference in total energy intake according to the presence of MetS in all groups, including single- and multiple-person households. However, as the eating habits of single-person households are generally poorer than those of multiple-person households ${ }^{11,16,17}$, additional studies are needed to evaluate eating habits such as eating behavior and the frequency of food intake, in addition to the high energy intake examined in the present study. The logistic regression analysis of this study, which corrected for BMI and household type variables, showed that participants with higher energy intake (approximately 1.5 times higher than the EER) had a $27 \%$ lower prevalence of low HDL-C and a $36 \%$ higher prevalence of high blood pressure. Our results were partly supported by a previous study of 7,081 men aged 30 years and older, in which those who overate more than four times weekly had a $141 \%$ higher prevalence of $\mathrm{MetS}^{18}$. The findings of this study confirmed that an average ratio of actual nutritional intake based on EES for middle-aged men $\geq 1.5$ or higher negatively impacted the components of MetS. The results also showed that a higher energy intake should be avoided to prevent MetS in middle-aged men.

Contrary to expectations, in this study, carbohydrate intake was lower in the MetS group than in the non-MetS group in both single- and multiple-person households. However, conflicting results have been reported regarding the relationship between MetS and carbohydrate intake. A study that compared the risk of MetS incidence by divid- ing male subjects into quintiles according to macronutrient intake reported a decreased risk of MetS with increased carbohydrate intake ${ }^{19}$. In contrast, a nutritional intake survey in middle-aged adults using data from the 2005 and 2007 KNHANES, according to the presence of MetS, showed a higher carbohydrate intake in the MetS group ${ }^{20}$. A 2018 cross-sectional study of 6,737 men and 8,845 women showed a proportionally increased risk of MetS with carbohydrate intake ${ }^{21}$. While the reasons for these conflicting results are unclear, excessive intake of carbohydrates, especially sugars, causes various diseases. Thus, we cannot conclude that significantly low carbohydrate intake would lead to MetS.

Analysis of PA level according to the presence of MetS showed lower levels of intermediate- and high-intensity leisure activities in the group of participants with MetS. Previous studies have reported that intermediate- and high-intensity PA reduced the incidence and risk of MetS ${ }^{22,23}$. The results of a study by Laaksonen et al. ${ }^{24}$ of 612 middle-aged men without MetS partially supported the findings of the present study, in which the risk of MetS was reduced by 45\% (OR: $0.55,95 \% \mathrm{CI}$; 0.31-0.98) among subjects who spent more time participating in leisure physical activities compared to the group that did not, after controlling for age and BMI.

Examination of PA level by household type according to the presence of MetS showed significantly lower PA levels for single-person households compared to multiple-person households in the group without MetS for intermediate-intensity PA. In contrast, single-person households showed significantly higher values than multiple-person households in the group with MetS. This finding is contrary to the hypothesis of a lower PA level in single-person households compared to multiple-person households, given the higher number of single-person households with MetS compared to single-person households without MetS. This study is the first to demonstrate differences in PA between single-and multiple-person households of middle-aged men depending on the presence of MetS. However, the total amount of PA did not differ according to the presence of MetS and household type; thus, additional studies are needed to examine the details of PA in daily life by narrowing the characteristics of single-person households (e.g., marital status, occupation) and using more objective measures of PA.

In this study, while the total amount of PA was lower in the group with MetS than in the group without MetS, the difference was not statistically significant. When the level of PA of the participants was further classified into four groups to analyze the risk of MetS, the prevalence of MetS was approximately $39 \%$ lower in those who were somewhat active (300-599 MET min/week) compared to those who were inactive (0-249 MET $\mathrm{min} /$ week), while the risk was significantly reduced by approximately $35 \%$ and $43 \%$ for active (600-1199 MET min/week) and very active $(\geq 1200$ MET min/week) levels of PA, respectively. The findings of this study confirmed that the risk of MetS was significantly lower for somewhat active (300-599 MET min/week) levels of PA. A prospective cohort study of the relationship 
between PA and cardiovascular disease observed a 30-40\% reduced risk of cardiovascular disease in middle-aged adults who were physically active ${ }^{25}$, consistent with the results of the present study. Sisson et al. ${ }^{26}$ also reported a $66 \%$ higher prevalence of MetS among men in the US with higher levels of sedentary behavior, which supported the finding that increasing total PA with regular exercise had a more potent positive effect on MetS ${ }^{27}$. In this study, abdominal obesity was 39\% lower in participants with somewhat active PA levels compared to baseline values. In addition, active levels of PA showed a $34 \%$ reduction in low HDL cholesterol levels compared to baseline values. Increased PA reportedly lowers the levels of LDL-cholesterol, a risk factor for cardiovascular disease, helps prevent cardiovascular disease by increasing HDL cholesterol levels, and has a positive effect on abdominal obesity ${ }^{4}$. The results of the present study demonstrated that PA aimed at active level (600-1199 MET $\mathrm{min} /$ week) could be effective in preventing MetS in middle-aged men.

This study has several limitations. First, as a cross-sectional study, it is difficult to verify the causal and temporal relationships among household type, energy intake, and PA level. Moreover, the ratio of single-person households is approximately $9.2 \%$ in this study. However, it was reported to be $30.2 \%$ of the total households in Korea ${ }^{5}$. Therefore, more data on single-person households should be collected in the near future. Second, compared to studies of the effects of income and education level on health-related behaviors, various socioeconomic variables such as financial status, education level, and marital status were inadequately corrected. Despite these limitations, these findings may increase the reliability of the results and could be generalized as a national trend based on the use of data from the KNHANES. Third, our data did not present detailed information on energy intake, such as eating habits and behaviors and micronutrient data. Therefore, further studies are needed to analyze detailed data on energy intake between single and multi-person households. In addition, this study has significance in that, unlike studies that analyzed elderly and female subjects, it was the first to analyze the PA level and energy intake according to household type and the presence of MetS in middle-aged men.

In conclusion, the results of this study showed that, although there was no difference in nutritional intake between single- and multiple-person households, the risk of MetS was significantly higher for the group of participants with at least 1.5 times the energy intake compared to that in the group with the lowest energy intake based on EER. No difference in the level of PA was observed between single- and multiple-person households; however, higher levels of PA, particularly among those who were only somewhat active (300-599 MET min/week), could have positive effects on the risk factors and prevalence of MetS regardless of household type. Therefore, we suggest reducing the total energy intake and increasing total PA to prevent MetS in middle-aged men regardless of household type is vital. With the recent increase in single-person households within the middle-aged population, more surveys on nutritional intake and
PA in middle-aged single-person households are needed, as poor health in middle age is likely to persist into old age.

\section{ACKNOWLEDGEMENTS}

This study analyzed the data provided by the Korean National Health and Nutrition Examination Survey 20162018, conducted by the Korea Centers for Disease Control and Prevention. This research was supported by the College of Education, Korea University Grant in 2021.

\section{REFERENCES}

1. Na DW, Jeong E, Noh EK, Chung JS, Choi CH, Park J. Dietary factors and metabolic syndrome in middle-aged men. J Agr Med Commun Health. 2010;35:383-94.

2. Bang SY, Hyeon SS. Comparison of physical activity and dietary patterns according to the degree of obesity in Korean men and women: data from the Seventh Korea National Health and Nutrition Examination Survey VII-1(2016). J DCS. 2018;19:1527-34.

3. Jung I, Kim JH. Sedentary behavior time in association with socioeconomic status, physical activity, and incidence of metabolic syndrome in male adults: based on the 7th (2016-2018) Korean national health and nutrition examination survey. KPEAW. 2020;34:83-99.

4. Park MY, Chung NN. Comparison of physical activity and nutrient intake according to abdominal obesity in their 20 s and 30 s in Korean men: data from the seventh Korea national health and nutrition examination survey VII (2016-2018). J Kor Soc Sports Sci. 2020;29:1113-25.

5. Statistics Korea. Causes of death statistics in 2019. KOSIS. 2020.

6. National statistical office. Population and housing census. KOSIS. 2020.

7. Lee K, Hwang Y, Ban HJ, Lim Sj, Jin HJ, Lee HS. The impact of the food market and policy tasks due to the increase in single-person households. KREI. 2015;1-241.

8. Lee KM, Lee KW, Hwang YS, Kang TH, Park YS, Jeong JM. Comparison of risk factors for type 2 diabetes mellitus in one-person households and multi-person households. Korean J Fam Pract. 2019;9:51-8

9. Kim MD, Park EO. Health behavior and metabolic syndrome of Korean adults in one-person households: based on the national cross-sectional survey. Health and Social Science. 2020;55:85101.

10. Cho KO. Associations between physical activity levels and health-related quality of life in the aged living alone. The Korean Journal of Physical Education. 2014;53:409-17.

11. Choi SB, Kwak JH, Chung HK, Kang HJ, Paik JK. Comparison of nutrient intake and metabolic syndrome between single person households and non-single person households in elderly subjects: from the sixth Korea national health and nutrition examination survey (KNHANES, 2013 2015). Korean J Food \& Nutr. 2020;33:32230.

12. Lee HB. Impact of physical activity level and types of activities of middle-aged women on risk factors of metabolic syndrome and energy metabolism. KJGD. 2014;22:371-80. 
13. Oh SW. Obesity and metabolic syndrome in Korea. Diabetes Metab J. 2011;35:561-6.

14. World Health Organization. Global physical activity questionnaire (GPAQ) analysis guide. WHO. 2012.

15. Kwak JH, Choi SB, Ju DJ, Lee MH, Paik JK. An analysis of the association between chronic disease risk factors according to household type for the middle-aged: the Korea national health and nutrition examination survey (2013 2015). Korean J Food \& Nutr. 2021;34:88-95.

16. Kim A. Effect of health behaviors, dietary habits, and psychological health on metabolic syndrome in one-person households among Korean young adults. J Digit Converg. 2018;16:493-509.

17. Heo YK, Sim KH. Dietary attitude of single households in metropolitan areas. Korean J Food \& Nutr. 2016;29:735-45.

18. Shin A, Lim SY, Sung J, Shin HR, Kim J. Dietary intake, eating habits, and metabolic syndrome in Korean men. J Am Diet Assoc. 2009;109:633-40.

19. Kim EY, Baek H. Association between metabolic syndrome and macronutrient intakes in adults aged 50 years and older based on the 2013-2017 Korean national health and nutrition examination survey data. J Korean Soc Food Cult. 2020;35:294-301.

20. Moon HK, Kong JE. Assessment of nutrient intake for middle aged with and without metabolic syndrome using 2005 and 2007 Korean national health and nutrition survey. Korean J Nutr. 2010;43:69-78.

21. Ha K, Kim K, Chun OK, Joung H, Song Y. Differential association of dietary carbohydrate intake with metabolic syndrome in the US and Korean adults: data from the 2007-2012 NHANES and KNHANES. Eur J Clin Nutr. 2018;72:848-60.

22. Tjonna AE, Lee SJ, Rognmo O, Stolen TO, Bye A, Haram PM, Loennechen JP, Al-Share QY, Skogvoll E, Slordahl SA, Kemi OJ, Najjar SM, Wisloff U. Aerobic interval training versus continuous moderate exercise as a treatment for the metabolic syndrome: a pilot study. Circulation. 2008;118:346-54.

23. Kim DI. The relationship between type of physical activity, frequency of physical activity per week, and metabolic syndrome risk factors among Korean adult population: data from the fifth Korea national health and nutrition examination survey V(2010-2012 years). J Kor Soc Sports Sci. 2015;24:1171-81.

24. Laaksonen DE, Lakka H-M, Salonen JT, Niskanen LK, Rauramaa $R$, Lakka TA. Low levels of leisure-time physical activity and cardiorespiratory fitness predict development of the metabolic syndrome. Diabetes Care. 2002;25:1612-8.

25. Kohl HW 3rd. Physical activity and cardiovascular disease: evidence for a dose response. Sports Med Health Sci. 2001;33:S47283.

26. Sisson SB, Camhi SM, Church TS, Martin CK, Tudor-Locke C, Bouchard C, Earnest CP, Smith SR, Newton RL, Rankinen T, Katzmarzyk PT. Leisure time sedentary behavior, occupational/ domestic physical activity, and metabolic syndrome in U.S. men and women. Metab Syndr Relat Disord. 2009;7:529-36.

27. Myers J, Kokkinos P, Nyelin E. Physical activity, cardiorespiratory fitness, and the metabolic syndrome. Nutrients. 2019;11:1652. 